\title{
Designer phase transitions in lithium-based spinels
}

DOE Award No. DE-FG02-07ER46381

Final report

\section{PI: Wouter Montfrooij \\ Department of Physics and Astronomy, University of Missouri, Columbia, 65203 573-639-0104, montfrooijw@ missouri.edu}

Date of report: September 12, 2011

Submitted to:

Dr. Thiyagarajan

Program Manager for X-Ray and Neutron Scattering The U.S. Department of Energy 


\section{Introduction / proposal summary}

\section{Introduction /original research scope}

When electrons in a metal become correlated with each other, new cooperative behavior can arise. This correlation is magnified when the metal has magnetic ions embedded in it. These atomic magnets try to line up with each other, but in doing so actually create a correlation between the motions of conduction electrons. In turn, these correlated electron motions prevent the magnetic ions from aligning, even at zero Kelvin. When this competition is strongest (at the so-called quantum critical point-QCP) the response of the system can no longer be described using the text book theory for metals. In addition, a range of new phenomena has been seen to emerge in the vicinity of a QCP, such as heavy-fermion superconductivity, coexistence of magnetism and superconductivity and hyper-scaling. The main goal of our research is to try to unravel the details of the feedback mechanism between electron motion and magnetism that lies at the heart of this new physics. We have chosen lithium-based spinel structures as the most promising family of systems to achieve our goal.

Known lithium-based spinels $\mathrm{Li}_{x} \mathrm{M}_{2} \mathrm{O}_{4}[\mathrm{M}=\mathrm{V}$, Ti and $\mathrm{Mn}]$ show a variety of ground states: heavy-fermion, superconducting, or geometrically frustrated local moment systems. $\mathrm{Li}_{\mathrm{x}} \mathrm{M}_{2} \mathrm{O}_{4}$ should be ideal systems for studying QCPs since their properties can easily be fine-tuned, simply by extracting some $\mathrm{Li}$ [which can be done without introducing disorder in the immediate surroundings of the magnetic ions]. The premise of the proposal was that since this Li-extraction can be done both in the metallic as well as in insulating compounds, that we can expand the types of quantum phase transitions that can be studied to beyond transitions in magnetic metals.

\section{The project called for developing a better understanding of quantum phase transitions by measuring all aspects of the electronic response of $\mathrm{Li}_{x} \mathrm{M}_{2} \mathrm{O}_{4}$ by means of neutron scattering, giving microscopic information about the behavior of the individual magnetic moments and their interactions, as well as by macroscopic measurements. In addition, the aim was to synthesize new lithium-based spinel compounds by using other transition metals that exhibit both $3^{+}$and $4^{+}$ valencies. Here we report on the progress we have made during the course of this grant both towards the stated goals and on new avenues that developed as a direct result of the data we collected during this grant.}


Project members during the course of the project

- Wouter Montfrooij, Assoc. Professor, Department of Physics

- Steven Keller, Assoc. Professor, Department of Chemistry, University of Missouri

- Thomas Heitmann [Post-doc partially supported FY10-FY11. Now FT position as instrument scientist at Missouri research reactor]

- Keary Schoen, [Post-doc till 8/31/07, supported by DOE under INIE project]

- Marcus Petrovic, [Master's student, fully supported, graduated May 2010]

- Jagat Lamsal [PhD student, 50\% supported, graduated Feb 2011]

- John Gaddy [graduate student, 100 \% supported, graduated Aug 2011]

- Mike Kraus [raduate student [Fall Semester 07, fully supported for this one semester]

- Alexander Schmets, Collaborator, Technical University of Delft, the Netherlands [visited for two months Oct-Dec 07, supported for travel and expenses]

- Lanika Ruzhitskaya [Graduate student, supported for 2 month to help with data rendition of percolation networks]

- Nathaniel Moore [Fall Semester 08, undergraduate student (senior), unsupported]

\section{Progress}

In this section we summarize the progress we have achieved on our DOE-funded research during the course of the grant, and how our findings relate to ongoing research.

\section{Progress towards stated goals}

Our project- "Designer phase transitions in lithium-based spinels" - called for developing a better understanding of quantum phase transitions by measuring all aspects of the electronic response of $\mathrm{Li}_{\mathrm{x}} \mathrm{M}_{2} \mathrm{O}_{4}$ by means of neutron scattering so as to obtain microscopic information about the behavior of the individual magnetic moments and their interactions, as well as by means of macroscopic measurements (resistance, specific heat, susceptibility). $\mathrm{M}$ in $\mathrm{Li}_{\mathrm{x}} \mathrm{M}_{2} \mathrm{O}_{4}$ stands for metals that can have both $3+$ and $4+$ valence states. In addition, the aim was to synthesize new lithium-based spinels.

Through extensive neutron scattering [I-III] and susceptibility [IV] studies of the (classical) insulator series $\mathrm{Li}_{\mathrm{x}} \mathrm{Mn}_{2} \mathrm{O}_{4}$ we were able to show that cluster reorientations account for E/Tscaling in quantum critical systems where uniform theories categorically exclude the existence of such scaling laws. This firmly established that geometry plays a part that cannot be ignored, as acknowledged by our peers [APS online journal "Physics"]. By the same token, it opens up a pathway towards reconciling intuitively appealing theories with experiments. Exploration of this pathway is the purpose of this renewal proposal. 
Our studies also showed that our original premise of studying a quantum phase transition in an insulating system could not be accomplished.
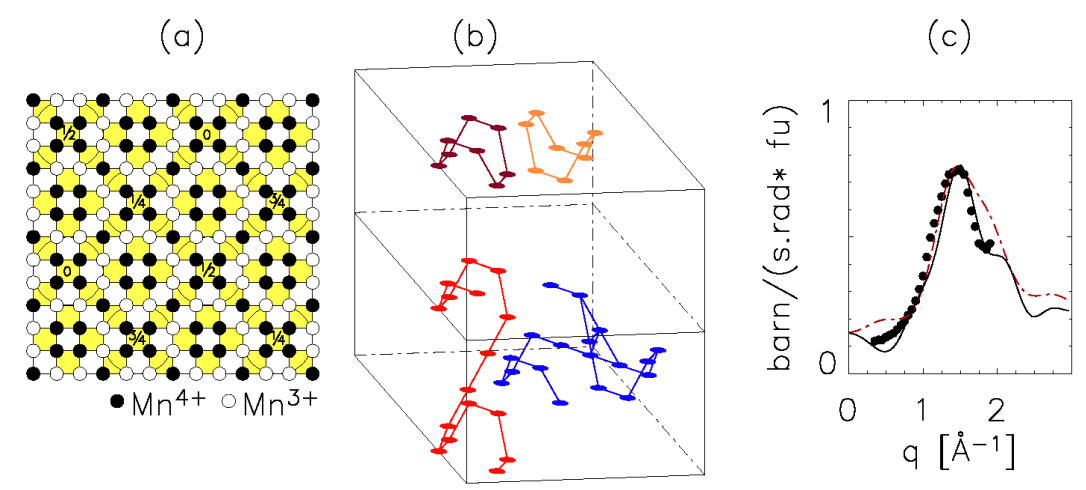

Fig 1: (a) The unit cell for stoichiometric $\mathrm{LiMn}_{2} \mathrm{O}_{4}$ projected along the c-axis [RodriguezCarvajal et al., Phys. Rev. Lett. 81 4660 (1998)]. The $\mathrm{Mn}^{4+}$ - ions (open circles) form 8-fold rings. (b) Two unit cells stacked along the c-axis for $\mathrm{LiMn}_{1.96} \mathrm{Li}_{0.04} \mathrm{O}_{4}$. Two ring clusters from part (a) are shown at the top of the figure. Upon Li substitution on the Mnsites some $\mathrm{Mn}^{3+}$-ions will become $\mathrm{Mn}^{4+}$-ions, leading to modified rings $[\mathrm{I}]$ and linked rings, two

possibilities of which are shown in the bottom half of the figure. (c) These 2-ring clusters give a satisfactory description of the neutron scattering data [I] (solid line, bottom left cluster in part b, or dashed line, bottom right cluster in part $b$.

While we established the existence of magnetic clusters in the off-the-shelf compound used in the battery industry (Fig. 1), we discovered that the quantum phase transition in this system was masked by a spin-glass transition (see Fig. 2) involving freezing out of these clusters. However, this ended up working in our favor as we could now make a direct comparison between cluster dynamics in quantum and classical systems, thereby showing E/T-scaling to be the hallmark of cluster reorientations in a classical system, and by extension, E/T-scaling in doped quantum critical systems also to be a hallmark of cluster reorientations. This is shown in Fig. 3.

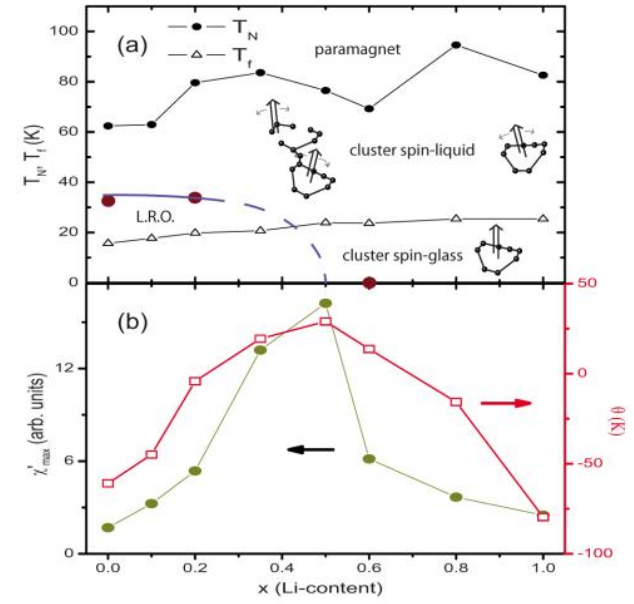

Fig. 2. (a) The phase diagram pertaining to spin cluster formation and freezing in $\mathrm{Li}_{\mathrm{x}} \mathrm{Mn}_{2} \mathrm{O}_{4}$ as determined from susceptibility and neutron scattering measurements ${ }^{\mathrm{IV}}$. The zero Kelvin order-disorder transition at $\mathrm{x}=0.5$ (blue line marked LRO) is masked by the formation of a cluster spin glass phase below $20 \mathrm{~K}$. Magnetic clusters are present above $20 \mathrm{~K}$, even in the presence of long range order (LRO). (b) The Weiss temperature $\theta$ (right axis) and the maximum in the uniform susceptibility (left axis) are plotted as a function of Li content demonstrating a peak near $\mathrm{x}=0.5$ as expected for an order-disorder transition.

While we have studied the $\mathrm{Li}_{\mathrm{x}} \mathrm{Mn}_{2} \mathrm{O}_{4}$ series to death, and are close [A] to solving the low 
temperature long-range magnetic structure of the stoichiometric parent compound $\mathrm{LiMn}_{2} \mathrm{O}_{4}$ that has 432 magnetic ions in its unit cell, we still have unfinished business when it comes to analyzing the neutron scattering and susceptibility data we took on $\mathrm{LiCoVO}_{4}, \mathrm{LiNiVO}_{4}$ and on these compounds with small Fe-substitution. While the analysis of these vanadates data ended up being low priority given our new discoveries [see below], we expect that the analysis will have been completed [C,D] fairly quickly with the aid of a new (IGERT funded) student of the PI.

Fig. 3 When I'(q,E,T)- the dynamic structure factor corrected for thermal population effects- is plotted on a log-log scale, the scattering appears to display dynamical scaling over the full range. The upper curve are the data for quantum critical $\mathrm{Ce}\left(\mathrm{Ru}_{1-\mathrm{x}} \mathrm{Fe}_{\mathrm{x}}\right)_{2} \mathrm{Ge}_{2}$ at $\mathrm{q}=4 \mathrm{~nm}^{-1}$. The lower curves are data ${ }^{\text {III }}$ for classical $\mathrm{LiMn}_{1.96} \mathrm{Li}_{0.04} \mathrm{O}_{4}$ taken at the Institute Laue-Langevin (lower) and at the University of Missouri (middle). The three curves are offset for plotting clarity. Each curve consists of many thousands of independent data points, showing that dynamical E/Tscaling is attributable to the presence of magnetic clusters.

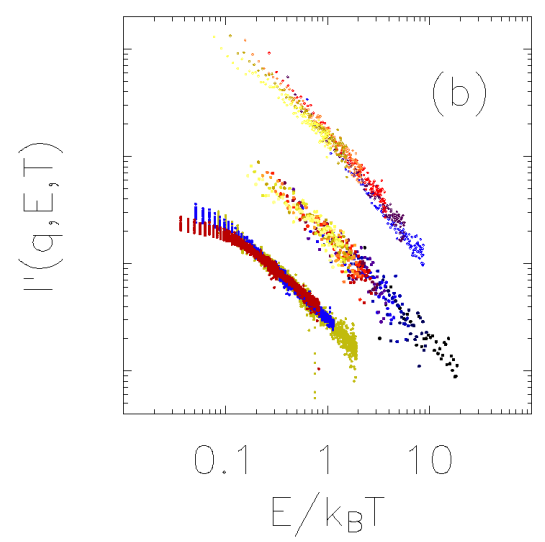

We failed in our aim of producing single phase Li-spinels based upon other elements. While we had some success in growing $\mathrm{LiRu}_{2} \mathrm{O}_{4}$, we were never able to grow it without impurity phases. We did not pursue this avenue further once it became clear that our classical insulators $\mathrm{Li}_{\mathrm{x}} \mathrm{Mn}_{2} \mathrm{O}_{4}$ were yielding results directly related to quantum critical physics.

\section{Progress on research not originally envisioned}

Our result on the lithium compounds, in correlation with our earlier work on 122-compounds told us that magnetic clusters become the entities that determine the response of doped quantum critical systems close to the QCP. This is a new insight as existing theories only deal with uniform systems (uniform in the sense that all magnetic lattice sites undergo an identical temperature evolution). We have made considerable progress in quantifying the influence of emerging magnetic clusters on the critical behavior of doped systems. We detail the progress in the following.

Based on our findings we have put forward a percolation-based phase diagram for quantum phase transitions in heavily doped compounds, shown in Fig. 4. While the standard phase 
diagram frequently shown in the literature explained the generic features of the systems that have been investigated, the problem with it was that it assumed that every ion on every lattice site undergoes an identical temperature evolution, something which no longer is a tenable proposition in a system that has been driven to the QCP through chemical doping, as we already demonstrated. The advantage of the new phase diagram is that is identifies the new structural units that should be, and can be, incorporated into existing theories.

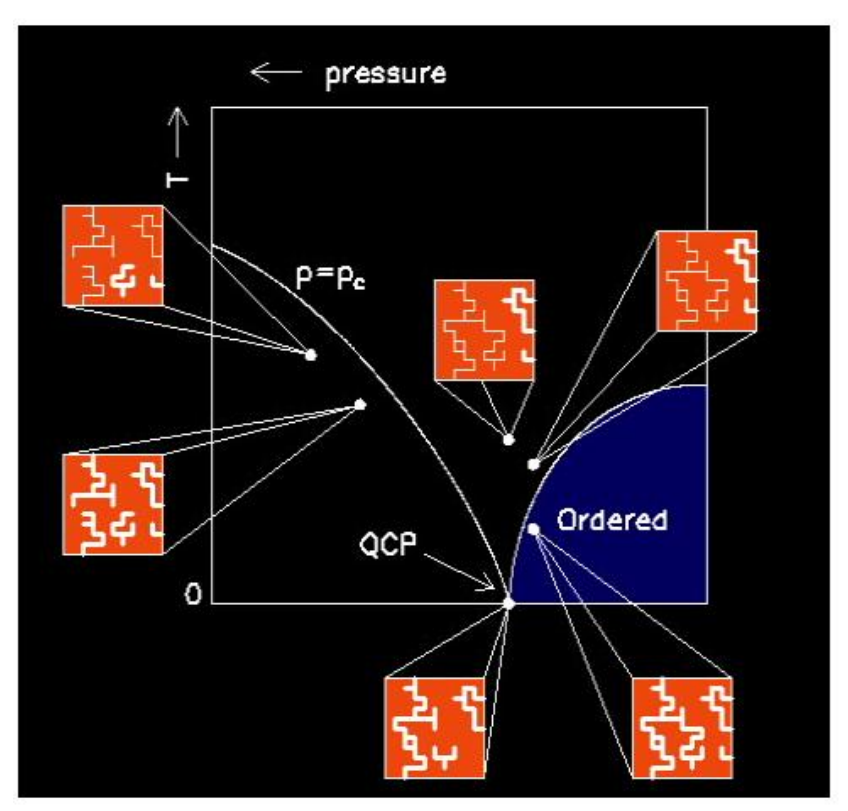

Fig. 4. Putative phase diagram for a quantum critical system subject to chemical disorder. A magnetically ordered phase (blue area bottom right) can be reached by cooling down a classical system (right vertical axis). Through lattice expansion or contraction, the transition temperature is driven down to $0 \mathrm{~K}$, the QCP. In the case of systems subject to chemical disorder, a distribution of Kondoshielding temperatures will result in the formation of magnetic clusters upon cooling because of the temperature dependent moment surviving probability p. The moments within isolated clusters will line up at a temperature inversely proportional to the size of the cluster (a straightforward quantum mechanical effect: the wavelength of the disordering fluctuation has to match the size of the cluster). This is shown in the inserts of the figure where the square box depicts the whole lattice, and the lines depict surviving neighboring moments. Thick lines indicate that the moments within such clusters have ordered, think lines indicate that the moments have not lined up yet because of thermal fluctuations. The lattice spanning cluster that exists above the percolation threshold $\left(p>p_{c}\right)$ connects the top to the bottom of the lattice. Once the moments within a cluster have lined up, they are less likely to suffer from Kondo shielding. In this picture, the QCP is the point where the lattice spanning cluster survives all the way down to zero Kelvin while maintaining its capability of ordering. This infinite cluster can be broken up by removing a single moment.

Next we studied whether the presence of clusters could (qualitatively) account for the measured specific heat data and neutron scattering results in quantum critical $\mathrm{Ce}\left(\mathrm{Ru}_{1-\mathrm{x}} \mathrm{Fe}_{\mathrm{x}}\right)_{2} \mathrm{Ge}_{2}$. The answer is yes [V, VI, E], and we show some results in Figs. 5 and 6. 


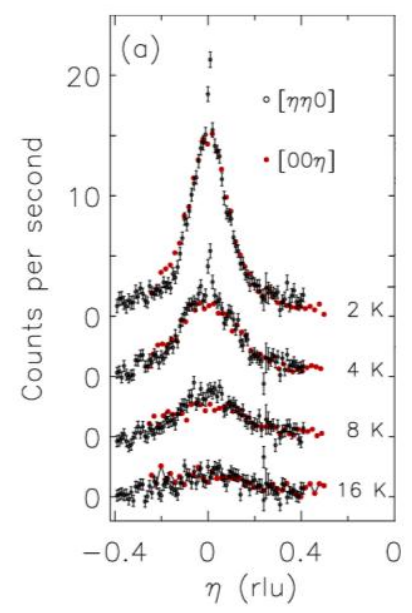

Fig. 5. Comparison between the emergence of short range order in experiments (left) and in simulations (right) in quantum critical $\mathrm{Ce}\left(\mathrm{Ru}_{1-\mathrm{x}} \mathrm{Fe}_{\mathrm{x}}\right)_{2} \mathrm{Ge}_{2}[\mathrm{E}]$. Both the shape as well as the quantitative T-dependence show good agreement. The T-dependence in the figure on the right depends on the temperature below which isolated clusters order because of finite size effects. The lower $\mathrm{T}$, the larger the cluster size for which its moments will line up, giving rise to new intensity appearing on top of intensity associated with smaller clusters that order at higher $\mathrm{T} . \eta=\mathrm{q}-\mathrm{q}_{\text {ord }}$.

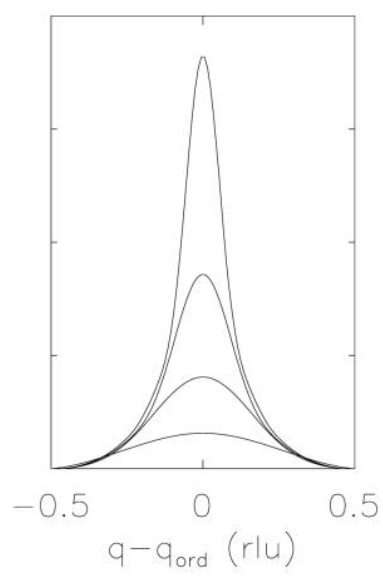

The good qualitative agreement between percolation-based calculations and the measured response of the system (Fig. 6) at these low temperatures demonstrates that cluster formation strongly influences the response.

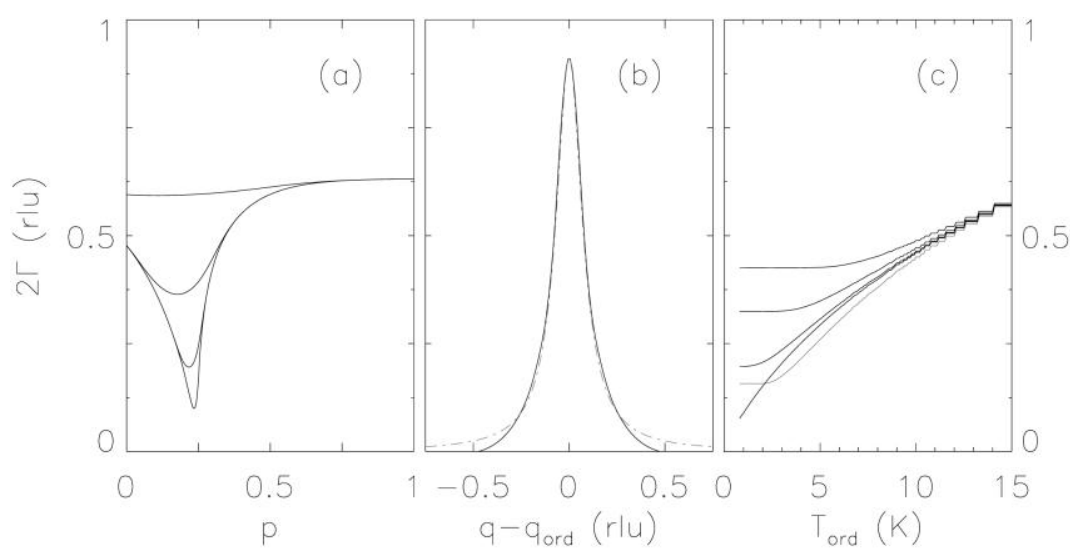

Fig.6. (a) Calculated $[\mathrm{E}]$ (inverse)
correlation length $\Gamma$ as a function
occupancy probability. $\mathrm{p}_{\mathrm{c}}=0.246$ is
the percolation threshold for this
body centered lattice. The four
curves have been calculated by
choosing the the maximum size of
the ordered cluster to be $\mathrm{N}=3$ (top
curve), $\mathrm{N}=30,300$ and 3000 (bottom

curve respectively); this mimics the temperature dependent finite size effects. (b) The calculated static structure factor at the percolation threshold (solid line) by summing over clusters with up to 3455 members. This reproduces the experimental findings at $\mathrm{T}=2 \mathrm{~K}$. The dashed line is the best fit to a Lorentzian line shape, explaining why the measured data showed a Lorentzian line shape in q where none was expected. (c) The calculated temperature dependence of the correlation length for various occupation probabilities. The temperature dependence has been linked to the maximum size $\mathrm{N}$ that any isolated cluster can have so that it order because of finite size effects: $\mathrm{N}=[2 \pi$ $\left.\mathrm{D} /\left(\mathrm{a} \mathrm{k} \mathrm{k}_{\mathrm{B}} \mathrm{T}\right)\right]^{3}$ with $\mathrm{D}=2 \AA / \mathrm{ps}$ the spin stiffness constant and a the length of the unit cell. The five curves are calculated for $\mathrm{p}=0.3$ (top), $0.27,0.253,0.246$ (bottom curve with respect to $\mathrm{T}=0$ intersect) and 0.21 (lowest curve except at the $\mathrm{T}=0$ intersect). The curve for $\mathrm{p}=0.246$ is indistinguishable from the experimentally determined correlation lengths for quantum critical $\mathrm{Ce}\left(\mathrm{Ru}_{1-\mathrm{x}} \mathrm{Fe}_{\mathrm{x}}\right)_{2} \mathrm{Ge}_{2}$. 
The same holds for the specific heat (not shown here) where an (R/4) $\ln 2$ contribution to the specific heat can be explained based upon clusters that peel off the infinite backbone upon lowering the temperature, and whose moments line up because of finite size effects. In fact, the specific heat upturn below $15 \mathrm{~K}$ in $\mathrm{Ce}\left(\mathrm{Ru}_{1-\mathrm{x}} \mathrm{Fe}_{\mathrm{x}}\right)_{2} \mathrm{Ge}_{2}$ is almost entirely due to these cluster effects [E]. Since standard theories do not incorporate such cluster effects in heavily doped systems, it is clear that the gap between theory and experiment can be closed considerably.

The qualitative behavior of the magnetic susceptibility in stoichiometric $\mathrm{YbRh}_{2} \mathrm{Si}_{2}$ under an applied magnetic field can also be understood on the basis of cluster formation [publication $\mathrm{V}$ and VII]. Altogether, these findings point the way towards new experiments that will lead to modified theories that take the fragmentation of the magnetic lattice upon cooling into account, and that should help us from separating true quantum critical physics resulting from quantum fluctuations from the effects attributable to changes in geometry.

In the process of relating quantum critical physics to cluster formation, we identified a new universality class in phase transitions based on percolation phenomena. In a nutshell, this new percolation model is similar to standard percolation, with the notable exception that only sites from the lattice spanning cluster can be removed. This reflects the fact that in quantum critical systems isolated clusters will have to line up because of finite size effects, and such ordered clusters are protected from Kondo shielding. This way, the isolated clusters will start to determine the critical behavior of the system. We have mapped this restricted percolation problem onto standard percolation problems [VI, VII], and the new critical exponents are listed in the table.

\begin{tabular}{|c|c|c|c|}
\hline Critical exponent & Standard percolation & Restricted percolation & relationship \\
\hline$\alpha$ & $\alpha, \beta, \gamma, v$ & $\alpha^{\prime}, \beta^{\prime}, \gamma^{\prime}, v^{\prime}$ & analytic \\
\hline$\beta$ & -0.62 & 1 & $2 \beta^{\prime}=\beta$ \\
\hline$\gamma$ & 0.41 & 0.205 & $\gamma^{\prime}=1-\beta$ \\
\hline$v$ & 1.8 & 0.595 & $v^{\prime}=1 / \mathrm{d}$ \\
\hline
\end{tabular}

It is noteworthy to remark that the critical exponent of the correlation length $(v)$ is below the limit of the Harris criterion, implying that disorder will be relevant in determining the critical behavior. Not only does this offer an explanation as to why doped quantum critical systems all appear to have different critical exponents, it also renders this restricted percolation model an 
ideal model for theorists to study the effects of impurities, and to study the transition from impurity dominated behavior, to regular critical behavior as predicted by the work of Chayes et al. (PRL 57, 2999 (1986)).

In summary, we believe that we have succeeded in developing a better understanding of quantum critical behavior, albeit not exactly following the route we had originally envisioned, and in our opinion, our work will end up resolving many of the outstanding issues related to quantum criticality.

\section{Cost status}

\begin{tabular}{|l|l|l|l|}
\hline Budget period & budget approved & $\begin{array}{l}\text { funds spent } \\
\text { (per 5/31/11) }\end{array}$ & unspent funds \\
\hline $6 / 1 / 07-5 / 31 / 08$ & $\$ 73,719$ & & \\
\hline $6 / 1 / 08-5 / 31 / 09$ & $\$ 70,827$ & & \\
\hline $6 / 1 / 09-5 / 31 / 10$ & $\$ 140,951$ & & \\
\hline Three year total & $\$ 285,487$ & $\$ 283,339.68$ & $\$ 2,157.32^{*}$ \\
\hline
\end{tabular}

*To be deobligated. It has come to light that certain health care benefits for one student had been collected twice over a period of two months. This mistake has been corrected, and the funds will be returned to the DOE ASAP. 


\section{Publications acknowledging current grant support}

I. J. Gaddy, J. Lamsal, M. Petrovic, W. Montfrooij, A. Schmets, and T. Vojta, Magnetic ordering in the spinel compound $\mathrm{Li}\left[\mathrm{Mn}_{2-x} \mathrm{Li}_{x}\right] \mathrm{O}_{4}(x=0,0.04)$, J. Appl. Phys. 105, 07D532 (2009).

II. J. Lamsal, J. Gaddy, M. Petrovic, W. Montfrooij, and T. Vojta, The search for quantum critical scaling in a classical system, J. Appl. Phys. 105, 07E322 (2009).

III. T. Heitmann, A. Schmets, J. Gaddy, J. Lamsal, M. Petrovic, T. Vojta, and W. Montfrooij, Magnetic excitations in the spinel compound $\mathrm{Li}_{x} \mathrm{Mn}_{1.96} \mathrm{Li}_{0.04} \mathrm{O}_{4}(x=0.2,0.6,0.8,1.0)$ : how a classical system can mimic quantum critical scaling, Phys. Rev. B 81, 014411 (2010) [editor's choice]

IV. Thomas Heitmann, John Gaddy, Jagat Lamsal, Marcus Petrovic, and Wouter Montfrooij. AC susceptibility of the quantum point mimicking series $\mathrm{Li}_{x} \mathrm{Mn}_{1.96} \mathrm{Li}_{0.04} \mathrm{O}_{4} \quad(x=0.0,0.1$, 0.2,0.35, 0.5, 0.6, 0.8, 1.0), J. Appl. Phys. 107, 09E143 (2010).

$\underline{\text { Submitted: }}$

V. Thomas Heitmann, John Gaddy, Jagat Lamsal, Thomas Vojta, and Wouter Montfrooij Comment on "Evidence for a Non-Fermi-Liquid Phase in Ge-Substituted $\mathrm{YbRh}_{2} \mathrm{Si}_{2}$ ", submitted to Phys. Rev. Lett., ArXiv:1012.3379

VI. Thomas Heitmann, John Gaddy, Jagat Lamsal, Thomas Vojta, and Wouter Montfrooij. Modified percolation theory and its application to quantum critical phenomena, submitted to Phys. Rev. B, Arxiv: 1101.6035.

VII. Tom Heitmann, John Gaddy and Wouter Montfrooij, Cluster formation in quantum critical systems, submitted to Journal of Physics, conference series, ArXiv:1109.5956

In Preparation:

A. J. Lamsal, J. Gaddy, T. Heitmann, and W. Montfrooij. The low temperature magnetic structure of stoichiometric $\mathrm{Li}_{x} \mathrm{Mn}_{2} \mathrm{O}_{4}$

B. J. Gaddy, T. Heitmann, T. Vojta, Y. Qiu, and W. Montfrooij, Cluster dynamics in the quantum critical compound $\mathrm{Ce}\left(\mathrm{Ru}_{0.24} \mathrm{Fe}_{0.76}\right)_{2} \mathrm{Ge}_{2}$, as measured by neutron scattering.

C. T. Heitmann, A. Schmets, J. Lamsal, J. Gaddy, and W. Montfrooij, Neutron scattering investigation of the microscopic dynamics of $\mathrm{LiCoVO}_{4}, \mathrm{LiNiVO}_{4}$ and the changes upon Fe-doping.

D. T. Heitmann, J. Lamsal, J. Gaddy, A. Schmets, and W. Montfrooij, AC susceptibility measurements of the long and short range magnetic order in $\mathrm{LiCoVO}_{4}, \mathrm{LiNiVO}_{4}$ and iron doped compounds.

E. John Gaddy, Thomas Heitmann, Jagat Lamsal, Thomas Vojta, and Wouter Montfrooij, Percolation induced quantum critical behavior in the doped heavy fermion system $\mathrm{Ce}\left(\mathrm{Ru}_{0.24} \mathrm{Fe}_{0.76}\right)_{2} \mathrm{Ge}_{2}$.

Theses:

i) Jagat Lamsal, Studies of electronic and magnetic properties of strongly correlated electron systems by neutron scattering and magnetization measurements, successfully defended $1 / 11$

ii) John Gaddy, Percolative Effects in Quantum Critical Systems: How Magnetic Clusters Shape the Response at Low Temperatures, successfully defended 8/11 


\section{Conference presentations:}

- APS08 March meeting: W. Montfrooij, Neutron scattering investigation of Lithium based spinels.

- MMM'08: W. Montfrooij, The search for quantum critical scaling in a classical system.

- MMM'08: J. Gaddy, Magnetic ordering in the spinel compound $\left.\mathrm{Li}_{[} \mathrm{Mn}_{2-x} \mathrm{Li}_{x}\right] \mathrm{O}_{4}(x=0,0.04)$.

- APS09: J. Gaddy, Monte Carlo calculations of the specific heat in quantum critical metals.

- MMM'10: T. Heitmann, AC susceptibility of the quantum point mimicking series $\mathrm{Li}_{x} \mathrm{Mn}_{1.96} \mathrm{Li}_{0.04} \mathrm{O}_{4}(x=0.0,0.1,0.2,0.35,0.5,0.6,0.8,1.0)$.

- Glass \& Optical Materials Division of the American Ceramic Society, Annual Meeting 2011, T. Heitmann, Cluster spin glass behavior in the Li-spinels, invited

- SCES 2011, Cambridge, UK. Tom Heitmann, John Gaddy, Jagat Lamsal and Wouter Montfrooij, The importance of magnetic cluster formation in quantum critical systems.

- APS Prairie section 2011 Iowa, Tom Heitmann, John Gaddy, Jagat Lamsal and Wouter Montfrooij. Magnetic clustering at a quantum critical point: A modified percolation theory

- APS March Meeting 2012, John Gaddy, Tom Heitmann, Jagat Lamsal, Wouter Montfrooij. Magnetic clustering at a quantum critical point: A modified percolation theory

\section{$\underline{\text { Colloquia and seminars }}$}

- Colorado State University: How a classical system can help us in solving the quantum critical riddle.

- Missouri Research Reactor seminar: Using TRIAX to investigate quantum critical phenomena.

- University of Wyoming: Percolation and quantum criticality: who wins?

\section{Peer press:}

Our research was picked up by the APS online journal physics in February 2010. Their writer Alex Klironomos wrote up the following précis of our work:

http://physics.aps.org/synopsis-for/10.1103/PhysRevB.81.014411

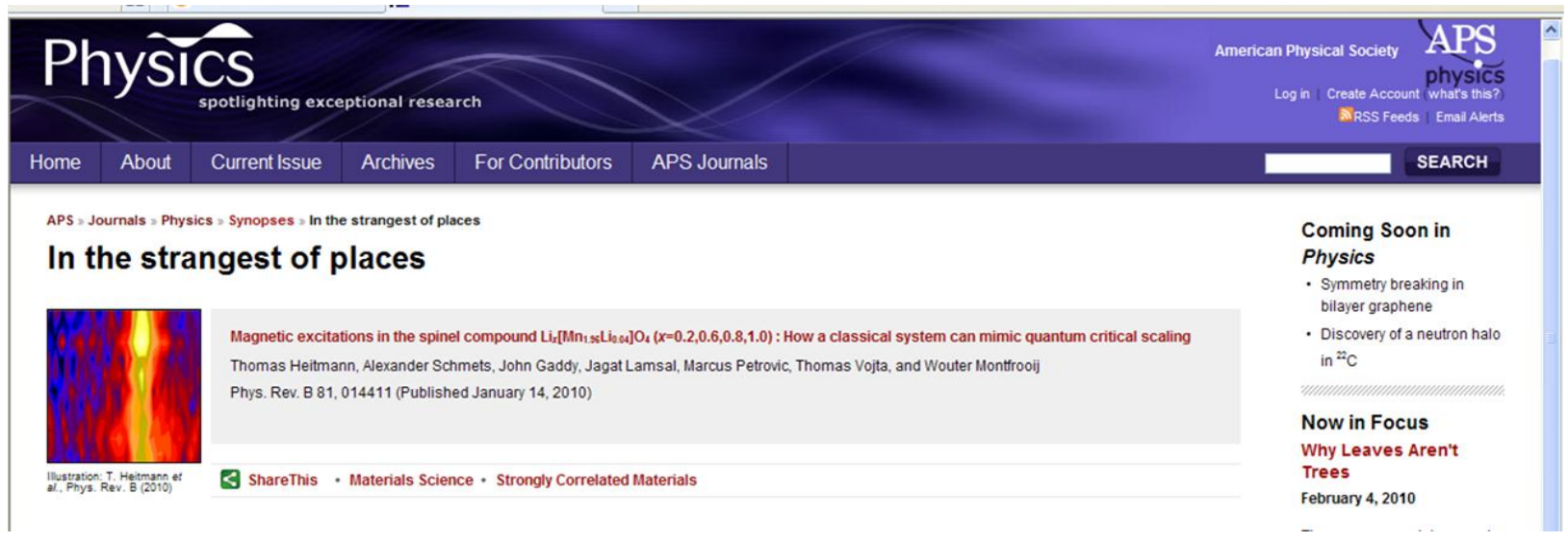

